\title{
Evolutionary Patterns of Gene Families Generated in the Early Stage of Vertebrates
}

\section{Yufeng Wang, Xun Gu}

Department of Zoology and Genetics, Center for Bioinformatics and Biological Statistics, Program of Bioinformatics and Computational Biology (BCB), Science II, Iowa State University, Ames, IA 50011, USA

Re: J Mol Evol (2000) 51:88-96. In Fig. 3A, the phylogeny of Activin $\beta(C, D)$ should be replaced as shown in the figure below. The discovery of frog Activin $\beta-\mathrm{D}$ indicates that the origin of Activin $\beta-C$ and $-D$ may occur after the tetrapod radiation. Thus, Activin-B should belong to three-cluster family (A, B, C/D/E), rather than Type I four-cluster. Nevertheless, this mis-

Correspondence to: X. Gu; email: xgu@iastate.edu classification does not change our major conclusion that at least two rounds of gene duplications in the early stage of vertebrates is a common pattern in gene family evolution.
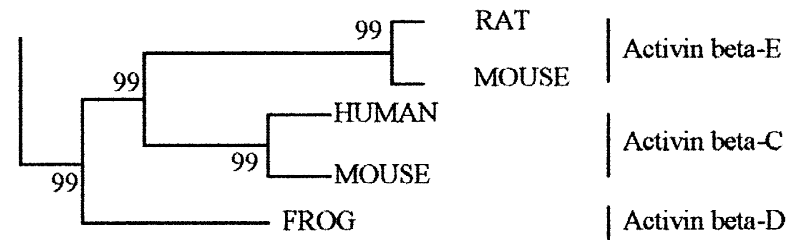